\title{
GRAPHIC FLOWS AND MULTIPLE DISJOINTNESS
}

\author{
BY
}

\author{
JOSEPH AUSLANDER AND NELSON MARKLEY
}

\begin{abstract}
A graphic flow is a totally minimal flow for which the only minimal subsets of the product flow are the graphs of the powers of the generating homeomorphism. The POD flows of Furstenberg, Keynes, and Shapiro [5] are examples of graphic flows. Graphic flows are in some ways analogous to ergodic systems with minimal self-joinings [11]. Various disjointness results concerning graphic flows and their powers are obtained, and their regular factors are determined.
\end{abstract}

When one forms the cartesian product of a minimal flow with itself, a natural question is to characterize the minimal subsets of the resulting flow. (The product flow cannot itself be minimal, since the diagonal is a closed invariant subset.) There are certain obvious minimal subsets, namely the graphs of the powers of the generating homeomorphism, which are all isomorphic to the original flow. In addition to these simply described minimal sets, the product of a flow with itself can, in general, contain a variety of more complicated minimal sets. As the term implies, the graphic minimal flows are essentially those for which the product flow contains only the intrinsic minimal subsets, namely the "graphs". This is analogous with Rudolph's idea of limiting the ergodic measures in the cartesian product of an ergodic measure-preserving system with itself; [11]. The seemingly mild definition of a graphic minimal flow has far-reaching consequences for minimal sets in cartesian products with more than two factors, as well as for products of the flows obtained by considering different powers of the homeomorphism.

The POD flows, defined by Furstenberg, Keynes and Shapiro [5], are examples of graphic minimal flows. The graphic minimal flows are themselves a subclass of the regular minimal flows, [1]. In these flows, the minimal subsets of the cartesian product are graphs of equivariant maps. Thus the automorphism group of the flow is a useful algebraic tool in the study of graphic minimal flows. Another is the Ellis group of a minimal flow. This group is important since any flow proximally equivalent to a graphic minimal flow is also graphic, and two proximally equivalent minimal flows also have the same Ellis groups.

The paper is in four sections. In the first section we review basic dynamical notions. In $\$ 2$ we define graphic minimal flows, and develop their basic properties. $\$ 3$ is devoted to disjointness properties. Our major result is that graphic minimal flows satisfy a "multiple disjointness" property (positive topological minimal selfjoinings). The final section deals with factors of products of powers of graphics.

Received by the editors July 30, 1984.

1980 Mathematics Subject Classification. Primary 54H20; Secondary 28D05.

(C)1985 American Mathematical Society $0002-9947 / 85 \$ 1.00+\$ .25$ per page 
1. Preliminaries. In this paper a flow will be a pair $(X, T)$ where $X$ is a compact Hausdorff space, and $T$ is a homeomorphism of $X$ onto $X$. We will usually but not always assume that $X$ is a metric space. We sometimes suppress the homeomorphism $T$ notationally, and just denote a flow by $X$. General references for the preliminary dynamical notions discussed in this section are [6] and [4]. (The latter should be used with care, since transformations are written on the right there.)

The trivial flow is the identity homeomorphism on the one-point space; in this case we write $X=1$.

If $\left(X_{i}, T_{i}\right)(i \in J)$ is a collection of flows, the product flow $\Pi_{i}\left(X_{i}, T_{i}\right)$ or $\left(\Pi_{i} X_{i}, \Pi_{i} T_{i}\right)$ is the flow on the product space $\Pi_{i} X_{i}$ defined by the action of each $T_{j}$ on the $j$ th coordinate; that is, the $j$ th coordinate of $\left(\Pi_{i} T_{i}\right)(x)$ is $T_{j}\left(x_{j}\right)$.

If $x \in X$, the orbit of $x$ is the set $\mathbf{O}(x)=\left\{T^{n}(x) \mid n \in \mathbf{Z}\right\}$. A subset $\Lambda$ of $X$ is a minimal set if $\Lambda$ is nonempty, closed, $T$-invariant $(T(\Lambda) \subset \Lambda)$, and contains no proper subsets with these properties. It is easy to see that a nonempty subset $\Lambda$ of $X$ is minimal if and only if it is the orbit closure of each of its points: $\Lambda=\overline{\mathbf{O}(x)}$, for all $x \in \Lambda$. If $\overline{\mathbf{O}(x)}$ is a minimal set, $x$ is said to be an almost periodic point. If $(X, T)$ is itself minimal, (so $X=\overline{\mathbf{O}(x)}$ for every $x \in X$ ), then we say that $(X, T)$ is a minimal flow.

If $(X, T)$ and $(Y, S)$ are flows, a homomorphism from $X$ to $Y$ is a continuous equivariant map $\pi$, that is, $\pi(T(x))=S(\pi(x))$, for all $x \in X$. If $(Y, S)$ is minimal, $\pi$ is necessarily onto. If there is a homomorphism from $X$ onto $Y$, we say that $Y$ is a factor of $X$ and $X$ is an extension of $Y$. The meaning of the terms "isomorphism" and "automorphism" should be clear. If $(X, T)$ is a minimal flow we write $A(X, T)$ or $A(X)$ for its group of automorphisms.

A flow $(X, T)$ is equicontinuous if the powers of $T\left\{T^{n} \mid n \in \mathbf{Z}\right\}$ form an equicontinuous family of maps. Every flow $(X, T)$ has a unique maximal equicontinuous factor-that is, there is an equicontinuous flow $(Y, S)$ which is a factor of $(X, T)$ such that every equicontinuous factor of $(X, T)$ is a factor of $(Y, S)[4]$.

The flow $(X, T)$ is (topologically) ergodic if the only open invariant set is $X$. When $X$ is metric this is equivalent to the existence of a point with a dense orbit (point transitive). When the flow $(X \times X, T \times T)$ is ergodic, $(X, T)$ is said to be (topologically) weak mixing. A minimal flow is weak mixing if and only if its maximal equicontinuous factor is trivial [8].

If $(X, T)$ is a flow, the points $x$ and $y$ in $X$ are proximal if there is a sequence $\left\{n_{i}\right\}$ such that $\lim T^{n_{i}}(x)=\lim T^{n_{i}}(y)$. (Here and elsewhere in the paper, when we use sequences in definitions and arguments we are assuming the phase space is metric. In case the space is not metric "sequences" should be replaced by "nets".) Equivalently, $x$ and $y$ are proximal if the diagonal $\Delta_{X} \subset \overline{\mathbf{O}(x, y)}$. We write $P(X, T)$ or just $P$ for the proximal relation: $P=\{(x, y) \mid x$ and $y$ are proximal $\}$. It is clear that $P$ is an invariant subset of the flow $(X \times X, T \times T)$ and that $P(X, T)=P\left(X, T^{n}\right)$ for $n \neq 0$.

It is also useful to relativize the proximal relation. A homomorphism $\pi: X \rightarrow Y$ is said to be proximal if, whenever $\pi(x)=\pi\left(x^{\prime}\right)$, then $\left(x, x^{\prime}\right) \in P$. In this case, we say that $X$ is a proximal extension of $Y$. 
We will need various facts about $\beta \mathbf{Z}$, the Stone-Čech compactification of the integers $\mathbf{Z}$, and its action on flows. Detailed proofs can be found in the cited references.

Let $V: \mathbf{Z} \rightarrow \mathbf{Z}$ by $V(n)=n+1$. Then $V$ extends to a homeomorphism of $\beta \mathbf{Z}$, which we still write as $V$. Note that the orbit of 0 under $V$ is $\mathbf{Z}$, which is dense in $\beta \mathbf{Z}$. Hence $(\beta \mathbf{Z}, V)$ is a point transitive flow. (The space $\beta \mathbf{Z}$ is compact Hausdorff, but is not metrizable.)

If $(X, T)$ is any flow, and $x$ is any point in $X$, then the map $n \rightarrow T^{n}(x)$ of $\mathbf{Z}$ to $X$ can be extended to a homomorphism of $(\beta \mathbf{Z}, V)$ to $(X, T)$, which we denote by $p \rightarrow p x(p \in \beta \mathbf{Z})$. The image of $\beta \mathbf{Z}$ under this homomorphism is precisely $\overline{\mathbf{O}(x)}$, so $p \rightarrow p x$ is onto if and only if the orbit $x$ is dense in $X$ ( $x$ is a transitive point). When $(X, T)=(\beta \mathbf{Z}, V)$ and $x=q \in \beta \mathbf{Z}$, the map $p \rightarrow p q$ defines an associative multiplication on $\beta \mathbf{Z}$. Thus $\beta \mathbf{Z}$ has a semigroup structure, in which multiplication is continuous in the first argument. That is, if $\left(p_{i}\right)$ is a net in $\beta \mathbf{Z}$ with $p_{i} \rightarrow p$ and $q \in \beta \mathbf{Z}$, then $p_{i} q \rightarrow p q$. In general, multiplication does not have any other continuity properties. Moreover, if $(X, T)$ is a flow and $x \in X$, the map $x \rightarrow p x(p \in \beta \mathbf{Z})$ is not continuous.

Since $\beta \mathbf{Z}$ is a compact Hausdorff space, $(\beta \mathbf{Z}, V)$ has minimal subsets, which coincide with the minimal left ideals (nonempty subsets $M$ such that $p M \subset M$, for $p \in \beta \mathbf{Z}$ ) of the semigroup $\beta \mathbf{Z}$. All of the minimal subsets of $\beta \mathbf{Z}$ are isomorphic. Moreover, they are universal minimal flows. Specifically, if $(X, T)$ is a minimal flow, then for any $x \in X$ the restriction of the map $p \rightarrow p x$ to a minimal set $M$ in $\beta \mathbf{Z}$ defines a homomorphism of $M$ onto $X$. Thus every minimal flow is a factor of $(M, V)$.

If $M$ is a minimal left ideal then $M$ contains idempotents (elements $u$ with $\left.u^{2}=u\right)$. If $(X, T)$ is a flow, then $x \in X$ is an almost periodic point if and only if $u x=x$, for some idempotent $u \in M$, and the points $x$ and $y$ of $X$ are proximal if and only if there is a minimal left ideal $M^{\prime}$ of $\beta \mathbf{Z}$ such that $p^{\prime} x=p^{\prime} y$ for all $p^{\prime} \in M^{\prime}$.

2. Graphic minimal flows. If $(X, T)$ is a minimal flow and $n \in \mathbf{Z}$, the graph of $T^{n}$ in the subset of $X \times X$ defined by $\Gamma_{n}=\left\{\left(x, T^{n}(x)\right) \mid x \in X\right\}$. Obviously, $\Gamma_{n}$ is a minimal subset of the product flow $(X \times X, T \times T)$ (in fact $\Gamma_{n}$ is isomorphic with $X)$. The minimal flow $(X, T)$ is called graphic if $(X, T)$ is totally minimal (i.e. $\left(X, T^{n}\right)$ is minimal for all $n \neq 0$ ) and the only minimal subsets of $X \times X$ are the graphs $\Gamma_{n}$. Equivalently, a totally minimal flow $(X, T)$ is graphic if every almost periodic point of $X \times X$ is of the form $\left(x, T^{n}(x)\right)$ for some $x \in X, n \in \mathbf{Z}$. It follows that the almost periodic points of the $n$-fold cartesian product flow $(X \times \cdots \times X$, $T \times \cdots \times T)$ are all of the form $\left(x, T^{k_{2}}(x), \ldots, T^{k_{n}}(x)\right)$, for some $x \in X$, $k_{2}, \ldots, k_{n} \in \mathbf{Z}$.

A useful characterization of graphic minimal flows is given by the following proposition. We omit the straightforward proof.

Proposition 1. A totally minimal flow $(X, T)$ is graphic if and only if, for all $x, y \in X$, there is an integer $n$ such that $\left(T^{n}(x), y\right) \in P$. 
If $(X, T)$ is graphic, then it is weakly mixing. In fact, if all almost periodic points of $X \times X$ are of the form $\left(x, T^{k}(x)\right)(x \in X, k \in \mathbf{Z})$, then any equicontinuous factor $(Y, S)$ must be finite. For, if $y, y^{\prime} \in Y$, then $\left(y, y^{\prime}\right)$ is an almost periodic point of $Y \times Y$ [4]. Let $\left(x, x^{\prime}\right)$ be an almost periodic point of $X \times X$ such that $x$ and $x^{\prime}$ map onto $y$ and $y^{\prime}$, respectively. Then $x^{\prime}=T^{k}(x)$, so $y^{\prime}=S^{k}(y)$, and $Y$ consists of a single orbit. Now an infinite minimal flow must be uncountable, so $Y$ is finite. If in addition $(X, T)$ is totally minimal, then $(Y, S)$ is totally minimal, so $Y$ is a singleton.

This argument also shows that a nontrivial equicontinuous minimal flow cannot be graphic.

In this connection, note that a weakly mixing flow is always totally minimal (since a nontotally minimal flow obviously has a nontrivial finite equicontinuous factor). Therefore, in the definition of graphic minimal flow we can replace "totally minimal" by "weakly mixing." That is, a minimal flow $(X, T)$ is graphic if and only if it is weakly mixing and the only almost periodic points in the product flow are the trivial ones $\left(x, T^{n}(x)\right)(x \in X, n \in \mathbf{Z})$.

If a minimal flow possesses the second property in the definition of graphic but is not necessarily totally minimal, then the next result shows that there is a canonically defined graphic subflow.

THEOREM 2. Let $(X, T)$ be a minimal flow and suppose the only almost periodic points of $(X \times X, T \times T)$ are of the form $\left(x, T^{k}(x)\right)(x \in X, k \in \mathbf{Z})$. Then there is a unique positive integer $m$ such that if $X_{0}=\operatorname{cl}\left\{T^{k m}\left(x_{0}\right) \mid k \in \mathbf{Z}\right\}$, then $\left(X_{0}, T^{m}\right)$ is graphic.

Proof. The preceding discussion shows that the maximal equicontinuous factor $(Y, S)$ of $(X, T)$ must be finite. Let $m$ be the cardinality of $Y, \pi: X \rightarrow Y, x_{0} \in X$, $y_{0}=\pi\left(x_{0}\right), y_{k}=S^{k}\left(y_{0}\right)(k=0,1, \ldots, m-1)$, so $Y=\left\{y_{0}, \ldots, y_{m-1}\right\}$. Then if $X_{0}=$ $\operatorname{cl}\left\{T^{k m}\left(x_{0}\right) \mid k \in \mathbf{Z}\right\}, X_{0}=\pi^{-1}\left(y_{0}\right), X=X_{0} \cup T\left(X_{0}\right) \cup \cdots \cup T^{m-1}\left(X_{0}\right)$, and this union is disjoint. We show $\left(X_{0}, T^{m}\right)$ is graphic. If $\left(X_{0}, T^{m}\right)$ is not totally minimal it has a nontrivial equicontinuous factor $\left(Y_{0}, S_{0}\right)$. In this case, it is easy to construct an equicontinuous factor $(X, T)$ of cardinality greater than $m$, which is a contradiction. Now suppose $x, x^{\prime} \in X_{0}$, with $\left(x, x^{\prime}\right)$ an almost periodic point of $\left(X_{0} \times X_{0}, T^{m} \times\right.$ $\left.T^{m}\right)$. Then $\left(x, x^{\prime}\right)$ is an almost periodic point of $(X \times X, T \times T)$, so $x^{\prime}=T^{r}(x)$ for some $r \in \mathbf{Z}$. But $T^{r}\left(X_{0}\right)=X_{0}$ if and only if $r$ is a multiple of $m$, so $x^{\prime}=T^{j m}\left(x_{0}\right)$, for some $j$, and $\left(X_{0}, T^{m}\right)$ is graphic.

Finally, we show that the positive integer $m$ is unique. For $l \in \mathbf{Z}$ write $\mathbf{O}_{l}(x)$ for the orbit of $x$ under $T^{l}$. If $\left(\overline{\mathbf{O}_{l}\left(x_{0}\right)}, T^{l}\right)$ is totally minimal, then $l$ must be a multiple of $m$. If not, then $\pi\left(\overline{\mathbf{O}_{l}\left(x_{0}\right)}\right)$ is not a singleton and some orbit of $\left(Y, S^{l}\right)$ is a nontrivial finite factor of $\left(\overline{\mathbf{O}_{l}\left(x_{0}\right)}, T^{l}\right)$. Therefore, if $l>0, l=j m$, where $j>0$. Since $\left(X_{0}, T^{m}\right)$ is totally minimal, $\overline{\mathbf{O}_{l}\left(x_{0}\right)}=\overline{\mathbf{O}_{j m}\left(x_{0}\right)}=\overline{\mathbf{O}_{m}\left(x_{0}\right)}=X_{0}$. Now $\left(X_{0}, T^{l}\right)=\left(X_{0}, T^{j m}\right)$ cannot be graphic if $j>1$, since $\left(x_{0}, T^{m}\left(x_{0}\right)\right)$ is an almost periodic point of $\left(X_{0} \times X_{0}, T^{l} \times T^{l}\right)$. This completes the proof. 
THEOREM 3. (i) Let $(X, T)$ be a graphic minimal flow, and let $(Y, S)$ be a minimal flow which is a factor of $(X, T), \pi:(X, T) \rightarrow(Y, S)$. Then $Y$ is trivial or $\pi$ is proximal.

(ii) Let $\pi:(X, T) \rightarrow(Y, S)$ be a proximal homomorphism of minimal flows. Then $(X, T)$ is graphic if and only if $(Y, S)$ is graphic.

Proof. (i) Suppose $\pi$ is not proximal. Then there are $x, x^{\prime} \in X$, with $x \neq x^{\prime}$, $\pi(x)=\pi\left(x^{\prime}\right)$ and $\left(x, x^{\prime}\right)$ is an almost periodic point of $(X \times X, T \times T)$. Since $(X, T)$ is graphic, $x^{\prime}=T^{m}(x)$ for some $m \neq 0$. So $\pi\left(T^{m}(x)\right)=\pi(x)$, and $(Y, S)$ is finite. Since $(X, T)$ is totally minimal, $\pi(z)=\pi(x)$ for all $z \in X$, so $Y$ is trivial.

(ii) Suppose $(X, T)$ is graphic. Let $\left(y, y^{\prime}\right)$ be an almost periodic point of $(Y \times Y, S \times S)$. Then there is an almost periodic point $\left(x, x^{\prime}\right)$ of $(X \times X, T \times T)$ with $\pi(x)=y, \pi\left(x^{\prime}\right)=y^{\prime}$. Since $(X, T)$ is graphic, $x^{\prime}=T^{k}(x)$, for some $k$, and so $y^{\prime}=S^{k}(y)$. Since total minimality is always preserved under homomorphisms, it follows that $(Y, S)$ is graphic.

Suppose $(Y, S)$ is graphic and $(X, T)$ is not totally minimal. Then there is a nonzero integer $k$ such that $\left(X, T^{k}\right)$ is not minimal. Since $\left(X, T^{k}\right)$ is pointwise almost periodic, there are distinct $T^{k}$ minimal subsets $X_{0}$ and $X_{1}$ of $X$. Then $\pi\left(X_{0}\right)=\pi\left(X_{1}\right)=Y$, because $(Y, S)$ is totally minimal. Let $y \in Y$. There exist $x_{0} \in X_{0}$ and $x_{1} \in X_{1}$ such that $\pi\left(x_{0}\right)=\pi\left(x_{1}\right)=y$. Now points on distinct minimal sets are never proximal, so $x_{0}$ and $x_{1}$ are not proximal (recall that $P(X, T)=$ $P\left(X, T^{k}\right)$ ). This contradicts the assumption that the homomorphism $\pi$ is proximal. If $\left(x, x^{\prime}\right)$ is an almost periodic point of $T \times T$, then $\left(y, y^{\prime}\right)$ is an almost periodic point of $S \times S$ where $y=\pi(x), y^{\prime}=\pi\left(x^{\prime}\right)$. Then $y^{\prime}=S^{k}(y)$, for some $k$, and it follows that $\pi\left(T^{k} x\right)=\pi\left(x^{\prime}\right)$. Now $\left(T^{k}(x), x^{\prime}\right)$ is almost periodic, and $\pi$ is proximal, so $x^{\prime}=T^{k}(x)$. This proves that $(X, T)$ is graphic.

Note that the above proof shows that total minimality is always preserved under proximal extensions.

We say that the minimal flows $(X, T)$ and $(Y, S)$ are proximally equivalent if they have a common proximal extension. (It is easy to see that this property is indeed an equivalence relation.) We also say that $(X, T)$ and $(Y, S)$ are in the same proximal class. The previous theorem shows that the property of being graphic depends only on the proximal class of the flow, and that graphic flows are "proximally prime"- the only factor of a graphic flow in a different proximal class is the trivial flow.

A minimal flow $(X, T)$ is regular if, whenever $x, x^{\prime} \in X$ with $\left(x, x^{\prime}\right)$ an almost periodic point of $X \times X$, there is an automorphism $\phi$ of $X$ such that $\phi(x)=x^{\prime}$. Clearly, a graphic minimal flow is regular. A number of alternate characterizations of regular minimal flows are given in [1].

If $(X, T)$ is regular and totally minimal, then $\left(X, T^{n}\right)$ is regular, for $n \neq 0$, since $\left(x, x^{\prime}\right)$ is almost periodic for $\left(X \times X, T^{n} \times T^{n}\right)$ if and only if it is almost periodic for $(X \times X, T \times T)$. (In particular, if $(X, T)$ is graphic, then $\left(X, T^{n}\right)$ is regular for $n \neq 0$.)

Suppose $(X, T)$ and $(Y, S)$ are minimal flows and $\pi: X \rightarrow Y$ is a homomorphism. If $\phi \in A(X, T)$ and $\psi \in A(Y, S)$ are such that $\pi \phi=\psi \pi$, we write $\psi=\tilde{\pi}(\phi)$. Then $\tilde{\pi}$ is a homomorphism of a subgroup $A_{\pi}$ of $A(X, T)$ to a subgroup of $A(Y, S)$. If $\pi$ is 
proximal, then $\tilde{\pi}$ is injective. (For if $\tilde{\pi}\left(\phi_{1}\right)=\tilde{\pi}\left(\phi_{2}\right)$, then $\pi\left(\phi_{1}(x)\right)=\pi\left(\phi_{2}(x)\right)$, $x \in X$ and $\left(\phi_{1}(x), \phi_{2}(x)\right)$ is both in $P$ and almost periodic for $T \times T$, so $\phi_{1}(x)=$ $\phi_{2}(x)$ and $\phi_{1}=\phi_{2}$.)

If $X$ is regular and $\psi \in A(Y, S)$, then, for $y \in Y,(y, \psi(y))$ is an almost periodic point of $Y \times Y$, and there is an almost periodic point $\left(x, x^{\prime}\right)$ for $T \times T$ with $\pi(x)=y$ and $\pi\left(x^{\prime}\right)=\psi(y)$. By regularity of $X$, there is a $\phi \in A(X, T)$ with $x^{\prime}=\phi(x)$ and it follows that $\psi=\tilde{\pi}(\phi)$. Therefore, $\tilde{\pi}\left(A_{\pi}\right)=A(Y, S)$. Similarly, if $Y$ is regular and $\phi \in A(X, T)$, there is a $\psi \in A(Y, S)$ such that $\tilde{\pi}(\phi)=\psi$; in this case $A_{\pi}=A(X, T)$.

Therefore, if both $X$ and $Y$ are regular, $A_{\pi}=A(X, T)$ and $\tilde{\pi}$ is a homomorphism of $A(X, T)$ onto $A(Y, S)$. In this context $\tilde{\pi}$ is an isomorphism if and only if $\pi$ is proximal. For if $\pi$ is proximal, then $\tilde{\pi}$ is one-to-one by the above discussion. If $\pi$ is not proximal, then there is an almost periodic point $\left(x, x^{\prime}\right)$ on $X \times X$ with $x \neq x^{\prime}$ and $\pi(x)=\pi\left(x^{\prime}\right)$. By regularity of $X, x^{\prime}=\phi(x)$, for some $\phi \in A(X, T)$ and $\tilde{\pi}(\phi)=\left.\mathrm{id}\right|_{Y}$. Thus $\phi$ is a nontrivial element in the kernel of $\tilde{\pi}$, and $\tilde{\pi}$ is not an isomorphism.

If $(X, T)$ is graphic, then as we noted earlier $\left(X, T^{n}\right)$ is regular for $n \neq 0$, and $A\left(X, T^{n}\right)=\mathbf{Z}$ ( $T$ is a generator)

This discussion immediately implies

THEOREM 4. Let $(X, T)$ be graphic, let $n \neq 0$, and let $(Y, S)$ be a regular factor of $\left(X, T^{n}\right)$. Then there is a $\sigma \in A(Y, S)$ such that $(Y, \sigma)$ is a factor of $(X, T)$ and $\sigma^{n}=S ;$ that is, $S$ is also a power of a graphic homeomorphism.

This theorem will be generalized later.

The following result will be used in $\S 4$.

THEOREM 5. Let $(X, T)$ and $(Y, S)$ be minimal flows with $(X, T)$ regular, and let $\pi: X \rightarrow Y$ be a homomorphism. Then $(Y, S)$ is regular if and only if, whenever $\alpha \in A(X, T)$, and $x, x^{\prime} \in X$ with $\pi(x)=\pi\left(x^{\prime}\right)$, then $\pi(\alpha(x))=\pi\left(\alpha\left(x^{\prime}\right)\right)$.

Proof. If $Y$ is regular, $\alpha \in A(X, T)$, and $\beta=\tilde{\pi}(\alpha)$, then $\pi(\alpha(x))=\beta(\pi(x))=$ $\beta\left(\pi\left(x^{\prime}\right)\right)=\pi\left(\alpha\left(x^{\prime}\right)\right)$. Conversely, if the condition holds, then there is a well-defined map $\alpha \rightarrow \tilde{\pi}(\alpha)$ from $A(X, T)$ to $A(Y, S)$ determined by $\tilde{\pi}(\alpha)(\pi(x))=\pi(\alpha(x))$. It follows easily that $(Y, S)$ is regular.

Furstenberg, Keynes, and Shapiro [5] defined a flow $(X, T)$ to be POD ("proximal orbit dense") if it is totally minimal, and if whenever $x$ and $y$ are in $X$ with $x \neq y$ there is an integer $n \neq 0$ such that $\left(T^{n}(x), y\right) \in P$. Hence by Proposition 1 , a POD flow is graphic. In order to pinpoint the relation between graphic and POD flows, we define a relation $L$ on $X$ by $L=\{(x, y) \in X \times X \mid \overline{\mathbf{O}(x, y)} \subset P\}$. The relation $L$, which is obviously invariant under $T \times T$, coincides with the syndetically proximal relation studied by Clay [3]. The following theorem gives several alternate characterizations of $L$.

THEOREM 6. Let $(X, T)$ be a minimal flow, and let $K=\bigcup\{I \mid I$ a minimal left ideal in $\beta \mathbf{Z}\}$. Then

(i) $L=\{(x, y) \mid q x=q y$, for all $q \in K\}=\left\{(x, y) \mid(x, y) \in P\right.$, and $\Delta_{X}$ is the only minimal subset of $\overline{\mathbf{O}(x, y)}\}$; 
(ii) if $(X, T)$ is graphic, and $x, y \in X$, then $(x, y) \in L$ if and only if $(x, y) \in P$ and $\left(T^{n}(x), y\right) \notin P$ for every $n \neq 0$.

Proof. (i) If $(x, y) \in L$, and $I$ is a minimal left ideal in $\beta \mathbf{Z}$, then $(q x, q y)$ $\in \overline{\mathbf{O}(x, y)} \subset P$, for $q \in I$. Let $I^{\prime}$ be a minimal left ideal for which $p^{\prime} q x=p^{\prime} q y$ for $p^{\prime} \in I^{\prime}$. Since $I^{\prime} q=I, q x=q y$ for all $q \in I$.

Suppose $q x=q y$ for all $q \in K$. Obviously, $(x, y) \in P$. If $\Gamma$ is a minimal subset of $\overline{\mathbf{O}(x, y)}$, then there is a minimal left ideal $I$ such that $(q x, q y) \in \Gamma$ for $q \in I$. Since $I \subset K, q x=q y$ and $\Gamma=\Delta_{X}$.

Suppose $(x, y) \in P$ and $\Delta_{X}$ is the unique minimal subset of $\overline{\mathbf{O}(x, y)}$. If $\left(x^{\prime}, y^{\prime}\right) \in$ $\overline{\mathbf{O}(x, y)}$, and $\Gamma^{\prime}$ in a minimal subset of $\overline{\mathbf{O}\left(x^{\prime}, y^{\prime}\right)}$, then $\Gamma^{\prime} \subset \overline{\mathbf{O}(x, y)}$, so $\Gamma^{\prime}=\Delta_{X}$ and it follows that $\left(x^{\prime}, y^{\prime}\right) \in P$. Therefore, $(x, y) \in L$.

(ii) Suppose $(X, T)$ is graphic. If $x, y \in X$ and $(x, y) \notin L$, then $q x \neq q y$ for $q$ in some minimal left ideal $I$ of $\beta \mathbf{Z}$. Now $(q x, q y)$ is an almost periodic point of $X \times X$, so $q x=T^{j}(q y)$ for some $j \neq 0 ;$ and $\left(T^{j}(q y), q y\right) \in \overline{\mathbf{O}(x, y)}, \quad(q y, q y)$ $\in \overline{\mathbf{O}\left(T^{-j},(x), x\right)}$, so $\left(T^{-j}(x), x\right) \in P$. On the other hand, if $(X, T)$ is any minimal flow (not necessarily graphic) and $x, y \in X$ with $\left(T^{n}(x), y\right) \in P$, then $\Gamma^{\prime}=$ $\left\{\left(T^{-n}\left(x^{\prime}\right), x^{\prime}\right) \mid x^{\prime} \in X\right\}$ is a minimal subset of $\overline{\mathbf{O}(x, y)}$. If also $(x, y) \in L$, then by the second characterization of $L$ in (i), $\Gamma^{\prime}=\Delta_{X}$ and $n=0$. This completes the proof.

Note that the first characterization of $L$ in Theorem 6 implies that $L$ is an equivalence relation. (This was also proved by Clay in [3].) The characterization of $L$ in the graphic case immediately implies

COROllary 7. A graphic flow is POD if and only if $L=\Delta$.

The relation $L$ can also be used to determine whether a graphic flow has a POD factor. In the next lemma, we write $P_{X}, L_{X}$ and $P_{Y}, L_{Y}$ for the $P$ and $L$ relations in $X$ and $Y$, respectively, $R_{\pi}$ for the relation defined by $\pi$, that is, $R_{\pi}=\left\{\left(x, x^{\prime}\right) \mid \pi(x)=\right.$ $\left.\pi\left(x^{\prime}\right)\right\}$, and $\hat{\pi}$ for the product homomorphism from $X \times X$ to $Y \times Y$ defined by $\hat{\pi}\left(x, x^{\prime}\right)=\left(\pi(x), \pi\left(x^{\prime}\right)\right)$.

LemMA 8. Let $(X, T)$ and $(Y, S)$ be minimal flows, and let $\pi: X \rightarrow Y$ be a proximal homomorphism. Then $R_{\pi} \subset L_{X}, \hat{\pi}^{-1}\left(P_{Y}\right)=P_{X}$ and $\hat{\pi}^{-1}\left(L_{Y}\right)=L_{X}$.

Proof. For any homomorphism, $\hat{\pi}\left(P_{X}\right) \subset P_{Y}$ and $\hat{\pi}\left(L_{X}\right) \subset L_{Y}$. By assumption, $R_{\pi} \subset P_{X}$. If $\left(x, x^{\prime}\right) \in R_{\pi}$ and $\left(z, z^{\prime}\right) \in \overline{\mathbf{O}\left(x, x^{\prime}\right)}$, then $\left(z, z^{\prime}\right) \in R_{\pi} \subset P_{X}$. That is, $\overline{\mathbf{O}\left(x, x^{\prime}\right)} \subset P_{X}$, and so $\left(x, x^{\prime}\right) \in L_{X}$, proving that $R_{\pi} \subset L_{X}$.

Next let $\left(x, x^{\prime}\right) \in \hat{\pi}^{-1}\left(P_{Y}\right)$. Then there is a $y_{0} \in Y$ with $S^{n_{i}}(\pi(x)) \rightarrow y_{0}$, $S^{n_{i}}\left(\pi\left(x^{\prime}\right)\right) \rightarrow y_{0}$ for some sequence $\left\{n_{i}\right\}$. Let (a subsequence of) $T^{n_{i}}(x) \rightarrow x_{0}$, $T^{n_{i}}\left(x^{\prime}\right) \rightarrow x_{0}^{\prime}$. Then $\pi\left(x_{0}\right)=\pi\left(x_{0}^{\prime}\right)=y_{0}$, and $\left(x_{0}, x_{0}^{\prime}\right) \in P_{X}$. Since $\left(x_{0}, x_{0}^{\prime}\right)$ $\in \overline{\mathbf{O}}\left(x, x^{\prime}\right)$, it follows that $\left(x, x^{\prime}\right) \in P_{X}$.

Finally, let $\left(x, x^{\prime}\right) \in \hat{\pi}^{-1}\left(L_{Y}\right)$ and $\pi(x)=y, \pi\left(x^{\prime}\right)=y^{\prime}$, then by what was just proved $\left(x, x^{\prime}\right) \in P_{X}$. If $\left(z, z^{\prime}\right) \in \overline{\mathbf{O}\left(x, x^{\prime}\right)}$, then $\left(\pi(z), \pi\left(z^{\prime}\right)\right) \in \overline{\mathbf{O}\left(y, y^{\prime}\right)}$ and $\left(\pi(z), \pi\left(z^{\prime}\right)\right) \in P_{Y}$ since $\left(y, y^{\prime}\right) \in L_{Y}$. Therefore, $\left(z, z^{\prime}\right) \in P_{X}$, so $\left(x, x^{\prime}\right) \in L_{X}$, and the proof is completed. 
Corollary 9 (Furstenberg, Keynes, Shapiro [5]). A POD flow is prime (has no nontrivial factors).

Proof. Suppose $X$ is POD, $\pi: X \rightarrow Y$ is a homomorphism, and $Y$ is not the trivial flow. Since $X$ is graphic, Lemma 8 applies, and $R_{\pi} \subset L_{X}=\Delta_{X}$, so $\pi$ is an isomorphism.

TheOREM 10. Let $(X, T)$ be a graphic minimal flow. Then $X$ has a nontrivial POD factor if and only if $L_{X}$ is closed (in which case $X / L_{X}$ is $P O D$ ).

Proof. If $(Y, S)$ is a POD factor of $(X, T)$, with $\pi: X \rightarrow Y$, then $L_{X}=\hat{\pi}^{-1}\left(L_{Y}\right)$ $=\hat{\pi}^{-1}\left(\Delta_{Y}\right)=R_{\pi}$, so $L_{X}$ is closed. Conversely, if $L_{X}$ is closed, and $Y=X / L_{X}$, then $L_{Y}=\hat{\pi}\left(L_{X}\right)=\Delta_{Y}$, so $Y$ is POD.

We conclude this section with some comments on examples of graphic flows. In [5], Furstenberg, Keynes, and Shapiro constructed an example of a POD (hence graphic) minimal flow. Also, del Junco [7] has shown that an example constructed by Chacon [2] is a POD. In the first example $(X, T)$ is isomorphic to $\left(X, T^{-1}\right)$, whereas in the second this is not the case; indeed $(X, T)$ and $\left(X, T^{-1}\right)$ are disjoint. (The definition and properties of disjointness will be presented in the next section, and the condition " $T$ disjoint from $T^{-1}$ " will play a role in some of the theorems there.)

The graphic flows can also be divided into two classes by whether or not they have a POD factor. The second author has constructed a graphic flow for which $L$ is not closed and hence by Theorem 10 does not have a POD factor. This is done by applying the Furstenberg, Keynes, Shapiro construction to a triangle in the surface of the torus instead of to an interval on the circle.

It is possible that a result of $\mathrm{Wu}[12$, Proposition 2.18] can also be applied to prove the existence of examples for which $L$ is not closed.

3. Disjointness and powers of graphics. There are two motivations for pursuing the theme of this section. First Furstenberg, Keynes, and Shapiro [5] proved that if a flow $(Y, S)$ was not an extension of a POD flow $(X, T)$, then $(Y \times X, S \times T)$ is minimal, that is, $(Y, S)$ and $(X, T)$ are disjoint. Keynes and Newton [9] pointed out that for $(X, T)$ POD $\left(X, T^{m}\right)$ and $\left(X, T^{n}\right)$ are disjoint when $m \neq 0, n \neq 0$, and $m \neq \pm n$. Then they proved that pairwise disjointness implied multiple disjointness for POD flows. In particular, $\left(X^{n}, T \times T^{2} \times \cdots \times T^{n}\right)$ is minimal. If the viewpoint is that POD flows are a special type of graphic minimal set, then one would like to know the extent to which these results hold for all graphic minimal sets.

The second motivation is our interest in investigating the topological analogues of Rudolph's concept of minimal self-joinings (MSJ) for measure-preserving transformations. The second author [10] has studied the following analogues of MSJ: The flow $(X, T)$ has topological minimal self-joinings (TSMJ) if it is graphic and if $\left(X^{n}, T^{a_{1}} \times \cdots \times T^{a_{n}}\right)$ is minimal whenever the $a_{i}$ are distinct nonzero integers. When the $a_{i}$ are restricted to distinct positive integers in the above definition, $(X, T)$ has positive topological minimal self-adjoinings (PTMSJ). (It is easy to see that these definitions are equivalent to those given in [10].) Simultaneously del Junco and Rudolph have been streamlining the MSJ concept. At present it is known that the 
original concept [11] holds if the defining condition can be verified for $\left(X^{k}, T \times\right.$ $\cdots \times T$ ) with $k>1$ which is now called $k$-fold MSJ. In other words, it is not necessary to verify the MSJ property for all $T^{a_{i}} \times \cdots \times T^{a_{k}}, a_{i} \neq 0$. Although 2-fold MSJ has many of the properties of MSJ, it is not known whether or not 2-fold MSJ implies $k$-fold. The graphic condition can be viewed as a topological analogue of 2-fold MSJ for which it is true that 2-fold implies $k$-fold. (See remarks following definition of graphic.) Consequently, it is very natural to ask if graphic implies either TMSJ or PTMSJ.

As in the previous section, let $M$ denote a fixed minimal left ideal in $\beta \mathbf{Z}$ and let $u$ be an idempotent in $M$. Then $p u=p$ for all $p \in M$ and $G=u M$ is a group with identity $u$. Fix $u$ and hence $G$. Given a flow $(X, T)$ and a point $x_{0} \in X$ such that $u x_{0}=x_{0}$, the Ellis group is defined by

$$
\mathbf{G}\left(X, T, x_{0}\right)=\left\{g \in G \mid g x_{0}=x_{0}\right\}
$$

which is obviously a subgroup of $G$. We will sometimes write $\mathbf{G}\left(X, x_{0}\right)$ for $\mathbf{G}\left(X, T, x_{0}\right)$. This group will play a crucial role in our study of the disjointness properties of graphic minimal sets. We begin by assembling the key facts about the Ellis group which will be needed later. For completeness a few more accessible but less well-known proofs are included.

It is quite obvious that if $Y$ is a minimal subset of $\Pi_{i}\left(X_{i}, T_{i}\right)$ and $u y=y$, then

$$
\mathbf{G}\left(Y, \prod_{i} T_{i}, y\right)=\bigcap_{i} \mathbf{G}\left(X_{i}, T_{i}, y_{i}\right)
$$

If $(X, T)$ is minimal and $x_{1}$ is another point in $X$ satisfying $u x_{1}=x_{1}$, then there exists $g \in G$ such that $x_{1}=g x_{0}$ and

$$
\mathbf{G}\left(X, T, x_{1}\right)=g \mathbf{G}\left(X, T, x_{0}\right) g^{-1} .
$$

Thus the conjugacy class of $\mathbf{G}\left(X, T, x_{0}\right)$ is an invariant of the isomorphism class of a minimal flow.

If $(X, T)$ is a regular minimal flow, and $x \in X$, then $\mathbf{G}(X, x)$ is a normal subgroup of $G$. Hence $\mathbf{G}(X, x)$ is independent of the point $x$, and in this case we just write $\mathbf{G}(X, T)$ or $\mathbf{G}(X)$.

The next two lemmas establish the relationship between these groups and proximal homomorphisms.

LEMMA 11. Let $(X, T),(Y, S)$ be minimal flows. Let $\pi: X \rightarrow Y$ be a homomorphism and let $x \in X, y \in Y$ with $\pi(x)=y$. Then

(i) $\mathbf{G}(X, x) \subset \mathbf{G}(Y, y)$,

(ii) $\pi$ is a proximal homomorphism if and only if $\mathbf{G}(X, x)=\mathbf{G}(Y, y)$.

Proof. [6, Chapter I, 4.1].

Lemma 12. Let $(X, T)$ and $(Y, S)$ be minimal flows, let $x \in X, y \in Y$ with $u x=x$, $u y=y$. Then $\mathbf{G}(X, x) \subset \mathbf{G}(Y, y)$ if and only if there is a minimal flow $\left(X^{\prime}, T^{\prime}\right)$ such that $X^{\prime}$ is a proximal extension of $X$, and $Y$ is a factor of $X^{\prime}$. 
Proof. If $X^{\prime}$ is as in the statement of the lemma, then $\mathbf{G}(X, x)=\mathbf{G}\left(X^{\prime}, x^{\prime}\right) \subset$ $\mathbf{G}(Y, y)$ by the previous lemma. Suppose $\mathbf{G}(X, x) \subset \mathbf{G}(Y, y)$ and let $X^{\prime}$ be the orbit closure of $(x, y)$ in $(X \times Y, T \times S)$. Let $\pi_{1}: X^{\prime} \rightarrow X$ and $\pi_{2}: X^{\prime} \rightarrow Y$ be the restrictions of the projection maps. Since $\mathbf{G}\left(X^{\prime},(x, y)\right)=\mathbf{G}(X, x) \cap \mathbf{G}(Y, y)=$ $\mathbf{G}(X, x), \pi_{1}$ is proximal by the previous lemma.

There is also a useful compact $T_{1}$ topology on $G$ called the $\tau$-topology. (Consult [4] or [6] for its definition and properties.) The groups $\mathbf{G}\left(X, T, x_{0}\right)$ are always $\tau$ closed. The relationship between the relative Stone-Čech topology on $M$ and the $\tau$-topology on $G$ is the following: If $\left\{\alpha_{i}\right\}$ is a net in $G$ which converges in $M$ to $p$, then $\alpha_{i}$ is convergent in the $\tau$-topology and its $\tau$-limit is $u$.

Two minimal flows $(X, T)$ and $(Y, S)$ are disjoint if $(X \times Y, T \times S)$ is minimal. The following lemma is well known, but a reference is not readily available, so we include a proof. (For a more general result, see [4, Proposition 18.7].)

Lemma 13. Let $(X, T)$ and $(Y, S)$ be minimal flows. Let $x \in X, y \in Y$ with $u x=x$, uy $=y$. Let $A=\mathbf{G}(X, x)$ and $B=\mathbf{G}(Y, y)$. Then $X$ and $Y$ are disjoint if and only if $A B=G$.

Proof. Suppose $A B=G$. If $g \in G, g=\alpha \beta$, for some $\alpha \in A, \beta \in B$, so $(x, g y)=$ $(\alpha x, \alpha \beta y)=(\alpha x, \alpha y)=\alpha(x, y) \in \overline{\mathbf{O}(x, y)}$. Therefore, $\{x\} \times G y \subset \overline{\mathbf{O}(x, y)}$, so $\{x\} \times Y \subset \overline{\mathbf{O}(x, y)}$ (since $\overline{G y}=Y$ ). It follows that $X \times Y=\overline{\mathbf{O}(x, y)}$ and so $X$ and $Y$ are disjoint. Conversely, suppose $X$ and $Y$ are disjoint, and let $g \in G$. Select $h \in G$ such that $h g(x, y)=(x, g y)$. Then $h g x=x$, so $\alpha=h g \in A$, and $\alpha y=h g y=$ $g y$, so $\alpha^{-1} g \in B$. Therefore, $g=\alpha\left(\alpha^{-1} g\right) \in A B$.

Lemma 14. Let $(X, T)$ be a minimal flow, let $x \in X$, and let $A=\mathbf{G}(X, x)$. Let $B$ be $a \tau$-closed subgroup of $G$. Suppose there is $a \beta \in B$, and an automorphism $\phi$ of $X$ for which the flow $(X, \phi)$ is minimal and $\beta x=\phi(x)$. Then $B A=G$.

Proof. Let $g \in G$. Since $(X, \phi)$ is minimal, there is a sequence $\left\{n_{i}\right\}$ such that $\phi^{n_{i}}(x) \rightarrow g x$. Now $\beta^{n_{i}} x=\phi^{n_{i}}(x)$, so $\beta^{n_{i}} x \rightarrow g x$. If $\beta^{n_{i}} \rightarrow q \in M$, then $\beta^{n_{i}} \stackrel{\tau}{\rightarrow} u q=\gamma$ [6, IX, Lemma 1.5] and $\gamma \in B$, since $B$ is $\tau$-closed. Now $q x=g x$, so $\gamma x=u q x=u g x$ $=g x$. Hence, $\gamma^{-1} g x=x$ and $\gamma^{-1} g \in A$. Hence, $g=\gamma^{-1} g \in B A$.

TheOREM 15. Let $(X, T)$ be a graphic minimal flow, let $A=\mathbf{G}\left(X, T^{m}, x\right)$ for some $m \neq 0$, and let $B$ be a $\tau$-closed subgroup of $G$. Then $B A=G$ if and only if $B$ is not contained in $A$.

Proof. Obviously $B A=G$ implies $B \not \subset A$. Let $\beta \in B \backslash A$. Then $(\beta x, x)$ is an almost periodic point of $\left(X \times X, T^{m} \times T^{m}\right)$ and $\beta x \neq x$. Therefore, $\beta x=T^{k}(x)$ for some $k \neq 0$, and the previous lemma applies.

COROllaRY 16. Let $(X, T)$ be a graphic minimal set and let $m$ be a nonzero integer. Then $A=\mathbf{G}\left(X, T^{m}, x_{0}\right)$ is a maximal $\tau$-closed normal subgroup of $G$.

Proof. We know $A$ is normal because $\left(X, T^{m}\right)$ is regular. If $B$ is a proper $\tau$-closed subgroup containing $A$, then $B A=B \neq G$. Then $B \subset A$ and $A=B$. 
COROllary 17. Let $(X, T)$ and $(Y, S)$ be minimal flows, with $(X, T)$ graphic, and let $m \neq 0$. Then either $\left(X, T^{m}\right)$ is disjoint from $(Y, S)$ or $\left(X, T^{m}\right)$ is a factor of a proximal extension $\left(Y^{\prime}, S^{\prime}\right)$ of $(Y, S)$.

Proof. Let $A=\mathbf{G}\left(X, T^{m}\right), B=\mathbf{G}(Y, S)$. If $\left(X, T^{m}\right)$ and $(Y, S)$ are not disjoint, then $B A \neq G$, so $B \subset A$ by Theorem 15 . The result now follows from Lemma 12 .

When $(X, T)$ is POD, this result can be strengthened. Specifically, if $(X, T)$ and $(Y, S)$ are not disjoint, then without loss of generality we can assume that $\left(Y^{\prime}, S^{\prime}\right)$ given by Corollary 17 is a minimal subset $Z$ of $(X \times Y, T \times S)$ and the projection $\pi_{2}$ of $Z$ onto $(Y, S)$ is proximal. If $(x, y)$ and $\left(x^{\prime}, y^{\prime}\right) \in Z$ with $x \neq x^{\prime}$, then because $(X, T)$ is POD there exists $\left(x_{0}, y_{0}\right) \in Z$ and $n \neq 0$ such that $\left(T^{n}\left(x_{0}\right), y_{0}\right) \in Z$. It follows that $X \times\left\{y_{0}\right\} \subset Z$ and $X \times Y=Z$, a contradicition. Therefore, $\pi_{2}$ is an isomorphism, and $(Y, S)$ is an extension of $(X, T)$. This was proved by Furstenberg, Keynes and Shapiro [5].

THEOREM 18. Let $(X, T)$ and $(Y, S)$ be graphic minimal flows and let $m$ and $n$ be nonzero integers.

(i) The flows $\left(X, T^{n}\right)$ and $\left(Y, S^{m}\right)$ are either disjoint or proximally equivalent.

(ii) Suppose $(X, T)$ and $(Y, S)$ are in different proximal classes. If $m \neq-n$, then $\left(X, T^{n}\right)$ and $\left(Y, S^{m}\right)$ are disjoint. If, in addition, $\left(X, T^{-1}\right)$ and $(Y, S)$ are in different proximal classes, then $\left(X, T^{n}\right)$ and $\left(Y, S^{m}\right)$ are disjoint for all $n, m \neq 0$.

Proof. (i) This follows from Theorem 15 and Lemma 12, applied successively to $\left(X, T^{n}\right)$ and $\left(Y, S^{m}\right)$.

(ii) Assume $\left(X, T^{n}\right)$ and $\left(Y, S^{m}\right)$ are not disjoint with $m$ and $n$ nonzero. It follows from Theorem 15 that $\mathbf{G}\left(X, T^{n}, x_{0}\right)=\mathbf{G}\left(Y, S^{m}, y_{0}\right)$. Let $Z$ be a minimal subset of $\left(X \times Y, T^{n} \times S^{m}\right)$, and let $\pi_{1}$ and $\pi_{2}$ be the projections of $Z$ onto $X$ and $Y$. By Lemma $11, \pi_{1}$ and $\pi_{2}$ are proximal homomorphisms of $\left(Z, T^{n} \times S^{m}\right)$ onto $\left(X, T^{n}\right)$ and $\left(Y, S^{m}\right)$, and consequently $\tilde{\pi}_{1}$ and $\tilde{\pi}_{2}$ are isomorphisms. In particular, $A(Z)=$ $A\left(Z, T^{n} \times S^{m}\right)$ is infinite cyclic.

Let $E$ and $F$ be the automorphisms of $\left(Z, T^{n} \times S^{m}\right)$ such that $\tilde{\pi}_{1}(F)=T$ and $\tilde{\pi}_{2}(E)=S$. It follows that $E$ and $F$ are generators of $A(Z), E^{m}=T^{n} \times S^{m}$, and $F^{n}=T^{n} \times S^{m}$. If $m \neq \pm n$, this is obviously impossible. If $m=n, E=F$, and $(Z, E)$ is a common proximal extension of both $(X, T)$ and $(Y, S)$ which contradicts the hypothesis. This proves the first statement. If $m=-n, E=F^{-1}$, and $(Z, E)$ is a proximal extension of $\left(X, T^{-1}\right)$ and $(Y, S)$ which is ruled out by the additional hypothesis of the second statement to complete the proof.

THEOREM 19. Let $(X, T)$ be a graphic flow and let $m$ and $n$ be nonzero integers. Then $\left(X, T^{m}\right)$ and $\left(X, T^{n}\right)$ are disjoint whenever $m \neq \pm n$. If $(X, T)$ and $\left(X, T^{-1}\right)$ are disjoint, then $\left(X, T^{m}\right)$ and $\left(X, T^{n}\right)$ are disjoint whenever $m \neq n$.

Proof. Essentially the same proof used in the preceding theorem gives the result. Specifically, replace $Y$ with $X$ and $S$ with $T$, and delete the one-sentence argument for the case $m=n$.

The next theorem is the main result of this section. 
THEOREM 20. Let $\left\{\left(X_{i}, T_{i}\right)\right\}, i \in J$, be a family of graphic minimal sets and for each $i \in J$ let $a(i)$ be a nonzero integer. If $\left(X_{i}, T_{i}^{a(i)}\right)$ and $\left(X_{j}, T_{j}^{a(j)}\right)$ are disjoint whenever $i \neq j$, then $\Pi_{i}\left(X_{i}, T_{i}^{a(i)}\right)$ is minimal.

Proof. First note that it suffices to establish the result for finite $J$ and that, without loss of generality, we can assume $a(i)>0$ for all $i \in J$. The proof proceeds by induction starting with $n=2$ which is part of the hypothesis. Assume the result holds when the cardinality of $J$ is $k \geqslant 2$ and suppose

$$
\prod_{i=1}^{k+1}\left(X_{i}, T_{i}^{a(i)}\right)
$$

is not minimal. We can also assume that $a(1) \leqslant a(2) \leqslant \cdots \leqslant a(k+1)$.

Let $x=\left(x_{1}, \ldots, x_{k+1}\right) \in \prod_{i=1}^{k+1} X_{i}$ such that $u x=x$. So $M x=Y$ is a proper minimal subset of $\prod_{i=1}^{k+1} X_{i}$. Denote the restriction of $\prod_{i=1}^{k+1} T_{i}^{a(i)}$ to $Y$ by $S$. Then

$$
\mathbf{G}(Y, S, x)=\bigcap_{i=1}^{k+1} \mathbf{G}\left(X_{i}, T_{i}^{a(i)}, x_{i}\right) .
$$

Because $\prod_{i=1}^{k+1}\left(X_{i}, T_{i}^{a(i)}\right)$ is not minimal by assumption, it follows from Theorem 15 and the induction assumption that

$$
\bigcap_{i=1}^{k} \mathbf{G}\left(X_{i}, T_{i}^{a(i)}, x_{i}\right) \subset \mathbf{G}\left(X_{k+1}, T_{k+1}^{a(k+1)}, x_{k+1}\right) \text {. }
$$

Therefore,

$$
\mathbf{G}(Y, S, x)=\bigcap_{i=1}^{k} \mathbf{G}\left(X_{i}, T_{i}^{a(i)}, x_{i}\right)
$$

and the projection $\pi_{k+1}$ of $(Y, S)$ onto $\prod_{i=1}^{k}\left(X_{i}, T_{i}^{a(i)}\right)$ is a proximal homomorphism. Similarly, the projections $\pi_{j}, j=1, \ldots, k$, of $(Y, S)$ onto $\prod_{i \neq j}\left(X_{i}, T_{i}^{a(i)}\right)$ are proximal homomorphisms.

Since both $(Y, S)$ and $\Pi_{i \neq j}\left(X, T_{i}^{a(i)}\right)$ are regular, the homomorphisms $\tilde{\pi}_{j}$ are isomorphisms of $A(Y, S)$ onto $A\left(\prod_{i \neq j}\left(X, T_{i}^{a(i)}\right)\right)$. Moreover, this latter group is precisely

$$
\left\{\prod_{i \neq j} T_{i}^{n(i)}: n(i) \in Z\right\}
$$

and is isomorphic to $\mathbf{Z}^{k}$. Let $E_{j}$ denote the element of $A(Y, S)$ such that $\tilde{\pi}_{k+1}\left(E_{j}\right)=$ $\Pi_{j=1}^{k} T^{\delta(i, j)}$ where $\delta(i, j)$ is 0 or 1 according as $i \neq j$ or $i=j$.

Since $\tilde{\pi}_{k+1}$ is an isomorphism, it follows that

$$
S=E_{1}^{a(1)} \circ \cdots \circ E_{k}^{a(k)} .
$$

Using projections and regularity it is easy to see that $E_{j}$ must be the restriction of

$$
I \times \cdots \times I \times T_{j} \times I \times \cdots \times I \times T_{k+1}^{\sigma(j)}
$$

to $Y$. (Here $I$ denotes the identity on $X_{i}$ for all $i$.) It now follows that

$$
a(k+1)=\sum_{j=1}^{k} \sigma(j) a(j)
$$

which will be needed later in the proof. 
The next major step is to show that $\sigma(j)= \pm 1$ for $1 \leqslant j \leqslant k$. If $\sigma(j)=0$, then $\tilde{\pi}_{j}\left(E_{j}\right)$ is the identity automorphism, which is impossible because $\tilde{\pi}_{j}$ is an isomorphism. Therefore, $\sigma(j) \neq 0$. To establish $|\sigma(j)| \leqslant 1$, we need some additional machinery.

Let $E \in A(Y, S)$. Then $E$ is a homeomorphism of $Y$ onto itself and we can consider the action of $\beta \mathbf{Z}$ on $Y$ determined by the flow $(Y, E)$ as well as by the flow $(Y, S)$. Since such considerations are essential, we use the notation $q(E)$ for this action of $q \in \beta \mathbf{Z}$ on $y \in Y$. (That is, if $\left\{n_{i}\right\}$ is a net in $\mathbf{Z}$ with $n_{i} \rightarrow q$, then $q(E) y=\lim E^{n_{i}}(y)$.) Note that

$$
\pi_{j}(q(E) y)=q\left(\tilde{\pi}_{j}(E)\right) \pi_{j}(y)
$$

Choose $w \in Y$ such that $u\left(E_{j}\right) w=w$. Hence for any $q \in \mathbf{G}\left(X, T_{j}, w_{j}\right)$,

$$
\begin{aligned}
\pi_{k+1}\left(q\left(E_{j}\right) w\right) & =q\left(\tilde{\pi}_{k+1}\left(E_{j}\right)\right) \pi_{k+1}(w) \\
& =q\left(\prod_{i=1}^{k} T_{i}^{\delta(i, j)}\right) \pi_{k+1}(w)=\pi_{k+1}(w) .
\end{aligned}
$$

Therefore, $\left(w, q\left(E_{j}\right) w\right) \in P_{S}$ for all $q \in \mathbf{G}\left(X_{j}, T_{j}, w_{j}\right)$. If $|\sigma(j)|>1$, then by Theorems 18 or 19 there exists $q \in \mathbf{G}\left(X_{j}, T_{j}, w_{j}\right) \backslash \mathbf{G}\left(X_{k+1}, T_{k+1}^{\sigma(j)}, w_{k+1}\right)$. Let $\pi$ be the projection of $Y$ onto $X_{k+1}$. Then $\pi\left(q\left(E_{j}\right) w\right)=q\left(T_{k+1}^{\sigma(j)}\right) w_{k+1} \neq w_{k+1}$. Since $\left(w, q\left(E_{j}\right) w\right) \in P_{S}, w_{k+1}$ and $\pi\left(\left(q\left(E_{j}\right) w\right)\right)$ are proximal for $T_{k+1}^{a(k+1)}$ and hence they are proximal for $T_{k+1}^{\sigma(j)}$. But this is impossible because $\left(w_{k+1}, q\left(T_{k+1}^{\sigma(j)}\right) w_{k+1}\right)$ is an almost periodic point for $T_{k+1}^{\sigma(j)} \times T_{k+1}^{\sigma(j)}$. Therefore, $\sigma(j)= \pm 1$ for $1 \leqslant j \leqslant k$.

Since $a(k+1)=\sum_{j=1}^{k} \sigma(j) a(j)$ and $a(1) \leqslant a(2) \leqslant \cdots \leqslant a(k+1)$, there exist at least two $j$ 's such that $\sigma(j)=1$. By rearranging the first $k$ coordinates we can assume $\sigma(1)=\sigma(2)=1$ and hence $E_{1} \circ E_{2}$ is the restriction of

$$
T_{1} \times T_{2} \times \cdots \times I \times T_{k+1}^{2}
$$

to $Y$.

We suppose now that $k=2$. If $T_{1}$ and $T_{3}$ are disjoint, then the invariance of $Y$ under $E_{1}=T_{1} \times I \times T_{3} \mid Y$ implies that $X_{1} \times\left\{x_{2}\right\} \times X_{3} \subset Y$. Applying $S^{k}$ yields $X_{1} \times\left\{T^{k a(2)} x_{2}\right\} \times X_{3} \subset Y$ for all $k$ in $\mathbf{Z}$ and hence $X_{1} \times X_{2} \times X_{3} \subset Y$, which is a contradiction. The same argument works using $E_{2}$ if $T_{2}$ and $T_{3}$ are disjoint. This leaves the case when $T_{1}, T_{2}$, and $T_{3}$ are all proximally equivalent. Because $Y$ is invariant under $E_{1} \circ E_{2}$, we can find a minimal subset $Y^{\prime}$ of $\left(X_{1} \times X_{2}, T_{1} \times T_{2}\right)$ which is contained in the projection of $Y$ onto $X_{1} \times X_{2}$. Clearly, $\left(Y^{\prime}, T_{1} \times T_{2}\right)$ and $\left(X_{1}, T_{1}\right)$ have the same Ellis group and are proximally equivalent. By Theorem 19, $\left(Y^{\prime}, T_{1} \times T_{2}\right)$ and $\left(X_{3}, T_{3}^{2}\right)$ are disjoint. Since $E_{1} \circ E_{2}=T_{1} \times T_{2} \times T_{3}^{2} \mid Y, Y^{\prime} \times X_{3}$ $\subset Y$. Let $\left(y_{1}, y_{2}\right) \in Y^{\prime}$. Applying $S^{k}$ to $\left\{\left(y_{1}, y_{2}\right)\right\} \times X_{3} \subset Y$ yields $\left\{\left(T_{1}^{k a(1)} y_{1}, T_{2}^{k a(2)} y_{2}\right)\right\} \times X_{3} \subset Y$ for all $k$. Because $\left(X_{1}, T_{1}^{a(1)}\right)$ and $\left(X_{2}, T_{2}^{a(2)}\right)$ are disjoint, we are again led to the contradiction $X_{1} \times X_{2} \times X_{3}=Y$.

Now suppose that $k>2$. If $T_{1}$ and $T_{2}$ are disjoint, the induction hypothesis tells us that $\left(X_{1} \times X_{2} \times X_{k+1}, T_{1} \times T_{2} \times T_{k+1}^{2}\right)$ is minimal, and since $Y$ is invariant under $E_{1} \circ E_{2}=T_{1} \times T_{2} \times I \times \cdots \times I \times T_{k+1}^{2} \mid Y, X_{1} \times X_{2} \times\left\{\left(x_{3}, \ldots, x_{k}\right)\right\} \times$ $X_{k+1} \subset Y$. The induction hypothesis also implies that $\left(X_{3} \times \cdots \times X_{k}, T_{3}^{a(3)} \times\right.$ $\left.\cdots \times T_{k}^{a(k)}\right)$ is minimal, so applying powers of $S$ we arrive at the contradiction 
$X_{1} \times X_{2} \times \cdots \times X_{k+1} \subset Y$. Finally, if $T_{1}$ and $T_{2}$ are not disjoint, they are proximally equivalent, so as in the proof of the corresponding part of the case $k=2$, $\left(Y^{\prime}, T_{1} \times T_{2}\right)$ is proximally equivalent with $\left(X_{1}, T_{1}\right)$. Hence, $\left(Y^{\prime}, T_{1} \times T_{2}\right)$ and $\left(X_{k+1}, T_{k+1}^{2}\right)$ are disjoint and $Y^{\prime} \times\left\{x_{3}, \ldots, x_{k}\right\} \times X_{k+1} \subset Y$. As before, we obtain $X_{1} \times X_{2} \times \cdots \times X_{k+1}=Y$, and the proof of the theorem is completed.

COROllary 21. Let $\left\{\left(X_{i}, T_{i}\right)\right\}_{i \in J}$ be a family of graphic minimal sets and for each $i \in J$ let $a(i)$ be a positive integer. If $\left(X_{i}, T_{i}\right)$ and $\left(X_{j}, T_{j}\right)$ are disjoint whenever $i \neq j$, then $\prod_{i}\left(X_{i}, T_{i}^{a(i)}\right)$ is minimal.

Corollary 22. Let $(X, T)$ be graphic.

(i) If $a(i), i \in J$, are positive integers such that $a(i) \neq a(j)$ when $i \neq j$, then $\Pi_{i}\left(X_{i}, T^{a(i)}\right)$ is minimal.

(ii) If $(X, T)$ and $\left(X, T^{-1}\right)$ are disjoint and $a(i), i \in J$, are nonzero integers such that $a(i) \neq a(j)$ when $i \neq j$, then $\prod_{i}\left(X_{i}, T^{a(i)}\right)$ is minimal.

COROllary 23. If $(X, T)$ is graphic, then $(X, T)$ has positive topological minimal self-joinings. If $(X, T)$ is graphic and disjoint from $\left(X, T^{-1}\right)$, then $(X, T)$ has topological minimal self-joinings.

4. Factors. In this section we study the regular factors of a cartesian product of pairwise disjoint powers of graphics. From our multiple disjointness theorem in the preceding section we know such a product flow is minimal. The main results are that the regular factors determine the proximal classes of all the factors and each regular factor is itself a cartesian product of factors of the coordinate flows.

Throughout this section $\left(X_{i}, T_{i}\right), i=1, \ldots, n$, will denote a finite number of minimal sets. (Repeats are not automatically being ruled out.) Let $k(i)$ be nonzero integers and let $(Z, U)$ be a factor of $\prod_{i=1}^{n}\left(X_{i}, T_{i}^{k(i)}\right)$. We say $(Z, U)$ is a factor of lower order if there exists a proper subset $F$ of $\{1, \ldots, n\}$ such that $(Z, U)$ is a factor of $\prod_{i \in F}\left(X_{i}, T_{i}^{k(i)}\right)$.

THEOREM 24. Suppose each $\left(X_{i}, T_{i}\right)$ is graphic, let $k(i), i=1, \ldots, n$, be nonzero integers such that $\left(X_{i}, T_{i}^{k(i)}\right)$ are pairwise disjoint, and let $\pi: \prod_{i=1}^{n}\left(X_{i}, T_{i}^{k(i)}\right) \rightarrow(Z, U)$ be a homomorphism. Then either $\pi$ is proximal or $(Z, U)$ is a factor of lower order. Therefore, if $(Z, U)$ is not the trivial flow, there exists a nonempty subset $F$ of $\{1, \ldots, n\}$ such that $(Z, U)$ is a proximal factor of $\prod_{i \in F}\left(X_{i}, T_{i}^{k(i)}\right)$.

Proof. If $\pi$ is not proximal, there exists an almost periodic point $\left(\left(x_{1}^{\prime}, \ldots, x_{n}^{\prime}\right),\left(x_{1}^{\prime \prime}, \ldots, x_{n}^{\prime \prime}\right)\right)$ such that $\pi\left(x_{1}^{\prime}, \ldots, x_{n}^{\prime}\right)=\pi\left(x_{1}^{\prime \prime}, \ldots, x_{n}^{\prime \prime}\right)$ and $\left(x_{1}^{\prime}, \ldots, x_{n}^{\prime}\right)$ $\neq\left(x_{1}^{\prime \prime}, \ldots, x_{n}^{\prime \prime}\right)$. Since $\left(x_{i}^{\prime}, x_{i}^{\prime \prime}\right)$ is an almost periodic point of $\left(X_{i} \times X_{i}, T_{i} \times T_{i}\right)$, $x_{i}^{\prime \prime}=T_{i}^{s(i)}\left(x_{i}^{\prime}\right)$ for some $s(i) \in \mathbf{Z}$ and for at least one $i, s(i) \neq 0$. Then

$$
\pi\left(T_{1}^{s(1)}\left(x_{1}^{\prime}\right) \times \cdots \times T_{n}^{s(n)}\left(x_{n}^{\prime}\right)\right)=\pi\left(x_{1}^{\prime}, \ldots, x_{n}^{\prime}\right)
$$

and $\pi \circ\left(T_{1}^{s(1)} \times \cdots \times T_{n}^{s(n)}\right)=\pi$ because $\prod_{i=1}^{n}\left(X_{i}, T_{i}^{k(i)}\right)$ is minimal and $T_{1}^{s(1)} \times$ $\cdots \times T_{n}^{s(n)}$ is one of its automorphisms. There are now two cases. 
Suppose $s(i) \neq 0, i=1, \ldots, n$. Then $\prod_{i=1}^{n}\left(X_{i}, T_{i}^{s(i)}\right)$ is weakly mixing and hence ergodic. Let $V_{1}$ and $V_{2}$ be open sets in $\prod_{i=1}^{n} X_{i}$ and let $S=\prod_{i=1}^{n} T_{i}^{s(i)}$. Then there exists $m$ such that $V_{1} \cap S^{m}\left(V_{2}\right) \neq \varnothing$. In particular, we can find $v \in V_{2}$ such that $S^{m}(v) \in V_{1}$. Since $\pi(v)=\pi\left(S^{m}(v)\right), \pi\left(V_{1}\right) \cap \pi\left(V_{2}\right) \neq \varnothing$ and $Z$ must be trivial.

If some $s(i)=0$, we may suppose $s(i) \neq 0$ for $1 \leqslant i \leqslant p$ and $s(p+1)=\cdots=$ $s(n)=0$ for some $p, 1 \leqslant p \leqslant n-1$. Now $S=S_{0} \times I$ where $S_{0}=\prod_{i=1}^{p} T_{i}^{s(i)}$ and $I$ is the identity on the remaining coordinates. If we write, for $z \in \prod_{i=1}^{n} X_{i}, z=(x, y)$ where $x \in \prod_{i=1}^{p} X_{i}$ and $y \in \prod_{i=p+1}^{n} X_{i}$, then $S(x, y)=\left(S_{0}(x), y\right)$. Since $S_{0}$ is weakly mixing, an argument as in the preceding paragraph implies $\pi(x, y)=\pi\left(x^{\prime}, y\right)$ for all $x, x^{\prime} \in \prod_{i=1}^{p} X_{i}$. It follows that $(Z, U)$ is a factor of $\prod_{i=p+1}^{n}\left(X_{i}, T_{i}^{k(i)}\right)$.

Corollary 25. If $\left(X_{i}, T_{i}\right)$ are graphic minimal sets such that $\left(X_{i}, T_{i}^{k(i)}\right), i=$ $1, \ldots, n$, are pairwise disjoint, then the factors of $\prod_{i=1}^{n}\left(X_{i}, T_{i}^{k(i)}\right)$ determine precisely $2^{n}$ distinct proximal classes.

Proof. If $E$ and $F$ are distinct subsets of $\{1, \ldots, n\}$, then $\prod_{i \in E}\left(X_{i}, T_{i}^{k(i)}\right)$ and $\prod_{i \in F}\left(X_{i}, T_{i}^{k(i)}\right)$ are in different proximal classes. (The trivial flow corresponds to $\varnothing$ and the identity homomorphism corresponds to $\{1, \ldots, n\}$.)

The following corollary is also a corollary of Proposition 3.3 in Keynes and Newton [9].

Corollary 26. Suppose each $\left(X_{i}, T_{i}\right)$ is POD and $k(i), i=1, \ldots, n$, are nonzero integers such that $\left(X_{i}, T_{i}^{k(i)}\right)$ are pairwise disjoint. Then every factor of $\prod_{i=1}^{n}\left(X_{i}, T_{i}^{k(i)}\right)$ is isomorphic to $\prod_{i \in F}\left(X_{i}, T_{i}^{k(i)}\right)$ for some $F \subset\{1, \ldots, n\}$.

Proof. Let $\pi: \prod_{i=1}^{n}\left(X_{i}, T_{i}^{k(i)}\right) \rightarrow(Z, U)$ be a homomorphism. By Theorem 24 it suffices to show that $\pi$ is an isomorphism when it is proximal. If $\pi$ is proximal and $\pi\left(x_{1}, \ldots, x_{n}\right)=\pi\left(x_{1}^{\prime}, \ldots, x_{n}^{\prime}\right)$, then it easily follows that $\left(x_{i}, x_{i}^{\prime}\right) \in L_{X_{i}}$ which equals $\Delta_{X_{i}}$ because $\left(X_{i}, T_{i}\right)$ is POD. Thus $\pi$ is one-to-one, and we are done.

Graphic minimal flows are not necessarily prime, so we cannot replace "POD" by "graphic" in Corollary 26. However, it is reasonable to ask whether the factors of a minimal $\prod_{i=1}^{n}\left(X_{i}, T_{i}^{k(i)}\right)$ with $\left(X_{i}, T_{i}\right)$ graphic are of the form $\prod_{i=1}^{n}\left(Z_{i}, U_{i}^{k(i)}\right)$. In fact, we show that every regular factor is of this form. Conversely, a factor of this form must be regular because (a) factors of graphics are graphic or trivial, (b) powers of graphics are regular, and (c) minimal subsets of products of regular minimal flows are regular. This characterizes only the regular factors. Since graphic minimal flows frequently have pairs of doubly aymptotic points, it is easy to construct nonregular factors by identifying the orbits of such pairs. In order to prove the characterization of regular factors we first prove a general theorem about regular minimal flows, which will then be applied to graphic minimal flows.

THEOREM 27. Suppose each $\left(X_{i}, T_{i}\right)$ is regular and the product flow $\prod_{i=1}^{n}\left(X_{i}, T_{i}\right)$ is minimal. If $(Z, U)$ is a regular minimal flow and if $\pi: \Pi_{i=1}^{n}\left(X_{i}, T_{i}\right) \rightarrow(Z, U)$ is a proximal homomorphism, then there exist regular minimal flows $\left(Z_{i}, U_{i}\right), i=1, \ldots, n$, such that, for each $i,\left(Z_{i}, U_{i}\right)$ is a factor of $\left(X_{i}, T_{i}\right)$ and $(Z, U)$ is isomorphic to $\prod_{i=1}^{n}\left(Z_{i}, U_{i}\right)$. 
Proof. Let $\alpha_{1}=\tilde{\pi}\left(T_{1} \times I \times \cdots \times I\right)$ and, for any $y \in \prod_{i=2}^{n} X_{i}$, define $\pi_{y}$ : $X_{1} \rightarrow Z$ by $\pi_{y}(x)=\pi(x, y)$. Clearly, $\pi_{y}$ is a homomorphism of $\left(X_{1}, T_{1}\right)$ to $\left(Z, \alpha_{1}\right)$ which is in general not onto. However, we can use Theorem 5 to show that its image $\left(\pi_{y}\left(X_{1}\right), \alpha_{1}\right)$ is regular. To do this, let $\phi \in A\left(X_{1}, T_{1}\right)$ and suppose $\pi_{y}(x)=\pi_{y}\left(x^{\prime}\right)$. Then $\pi(x, y)=\pi\left(x^{\prime}, y\right)$ and because both $\prod_{i=1}^{n}\left(X_{i}, T_{i}\right)$ and $(Z, U)$ are regular,

$$
\begin{aligned}
\pi_{y}(\phi(x)) & =\pi(\phi(x), y)=\tilde{\pi}(\phi \times I) \pi(x, y) \\
& =\tilde{\pi}(\phi \times I) \pi\left(x^{\prime}, y\right)=\pi\left(\phi\left(x^{\prime}\right), y\right)=\pi_{y}\left(\phi\left(x^{\prime}\right)\right) .
\end{aligned}
$$

Let $R_{y}$ denote the equivalence relation determined by $\pi_{y}$. The crucial step is to show that $R_{y}=R_{y^{\prime}}$ for all $y$ and $y^{\prime}$. Note that there is also a homomorphism $\pi_{x}$ from the minimal flow $\prod_{i=2}^{n}\left(X_{i}, T_{i}\right)$ to $(Z, S)$ where $S=\alpha_{2} \circ \cdots \circ \alpha_{n}$ and $\alpha_{i}=$ $\tilde{\pi}\left(I \times \cdots \times T_{i} \times \cdots \times I\right)$. If $\left(x, x^{\prime}\right) \in R_{y}$, then $\pi(x, y)=\pi\left(x^{\prime}, y\right), \pi_{x}(y)=$ $\pi_{x^{\prime}}(y)$, and $\pi_{x}=\pi_{x^{\prime}}$. (Two homomorphisms defined on the same minimal flow are equal if they agree at one point.) Now $\pi\left(x, y^{\prime}\right)=\pi\left(x^{\prime}, y^{\prime}\right)$ for all $y^{\prime}$ and $\left(x, x^{\prime}\right) \in R_{y^{\prime}}$ for all $y^{\prime}$. It follows that $R_{y}=R_{y^{\prime}}$ for all $y$ and $y^{\prime}$ and we let $R_{1}$ denote this equivalence relation. Similarly, there is such an equivalence relation $R_{i}$ for each $i$, Let $\left(Z_{i}, U_{i}\right)=\left(X_{i}, T_{i}\right) / R_{i}$ and note that $\left(Z_{i}, U_{i}\right)$ is regular because it is isomorphic to the regular minimal flow $\left(\pi\left(X_{i}\right), \alpha_{i}\right)$.

It remains to show that $R_{\pi}=\prod_{i=1}^{n} R_{i}$, i.e. $\pi\left(x_{1}, \ldots, x_{n}\right)=\pi\left(x_{1}^{\prime}, \ldots, x_{n}^{\prime}\right)$ if and only if $\left(x_{i}, x_{i}^{\prime}\right) \in R_{i}$ for $i=1, \ldots, n$. First suppose $\pi\left(x_{1}, \ldots, x_{n}\right)=\pi\left(x_{1}^{\prime}, \ldots, x_{n}^{\prime}\right)$. Let $y=\left(x_{2}, \ldots, x_{n}\right)$ and $y^{\prime}=\left(x_{2}^{\prime}, \ldots, x_{n}^{\prime}\right)$. Then $\pi_{y}\left(x_{1}\right)=\pi_{y^{\prime}}\left(x_{1}^{\prime}\right)$, and $x_{1}$ and $x_{1}^{\prime}$ are proximal. It follows that $\pi_{y}\left(x^{\prime \prime}\right)=\pi_{y^{\prime}}\left(x^{\prime \prime}\right)$ for some $x^{\prime \prime} \in X$ and $\pi_{y}=\pi_{y^{\prime}}$. Thus, $\pi_{y}\left(x_{1}\right)=\pi_{y}\left(x_{1}^{\prime}\right)$ and $\left(x_{1}, x_{1}^{\prime}\right) \in R_{1}$. Likewise, $\left(x_{i}, x_{i}^{\prime}\right) \in R_{i}$ for $i=2, \ldots, n$. Now suppose $\left(x_{i}, x_{i}^{\prime}\right) \in R_{i}$ for $i=1, \ldots, n$. Because $R_{i}=R_{y}$ for all $y \in \prod_{j \neq i} X_{i}$, $\pi\left(x_{1}, \ldots, x_{n}\right)=\pi\left(x_{1}^{\prime}, x_{2}, \ldots, x_{n}\right)=\pi\left(x_{1}^{\prime}, x_{2}^{\prime}, x_{3}, \ldots, x_{n}\right)=\cdots=\pi\left(x_{1}^{\prime}, \ldots, x_{n}^{\prime}\right)$, and the proof is complete.

THEOREM 28. Suppose each $\left(X_{i}, T_{i}\right)$ is graphic and $k(i), i=1, \ldots, n$, are nonzero integers such that $\left(X_{i}, T_{i}^{k(i)}\right)$ are pairwise disjoint. Let $(Z, U)$ be a factor of $\prod_{i=1}^{n}\left(X_{i}, T_{i}^{k(i)}\right)$. Then $(Z, U)$ is a regular minimal set if and only if there exist factors $\left(Z_{i}, U_{i}\right)$ of $\left(X_{i}, T_{i}\right), i=1, \ldots, n$, such that $(Z, U)$ is isomorphic to $\prod_{i=1}^{n}\left(Z_{i}, U_{i}^{k(i)}\right)$. Furthermore, when $(Z, U)$ is a regular minimal set, the following hold:

(a) The homomorphism $\pi$ is proximal if and only if none of the $\left(Z_{i}, U_{i}\right)$ is trivial.

(b) The rank of the kernel of $\tilde{\pi}$ equals the number of $i$ 's for which $\left(Z_{i}, U_{i}\right)$ is trivial.

Proof. The "if" part follows from remarks preceding Theorem 27. For the "only if" part we can by Theorem 24 assume $(Z, U)$ is a proximal factor and then apply Theorems 27 and 4 . This proves the characterization of the regular factors.

Assuming $(Z, U)$ is regular there exists a homomorphism $\pi: \prod_{i=1}^{n}\left(X_{i}, T_{i}^{k(i)}\right) \rightarrow$ $\prod_{i=1}^{n}\left(Z_{i}, U_{i}^{k(i)}\right)$, and for each $i$ there exists a homomorphism $\pi_{i}:\left(X_{i}, T_{i}\right) \rightarrow\left(Z_{i}, U_{i}\right)$. Because two homomorphisms from one regular minimal set to another differ by an automorphism of the image flow, we can assume without loss of generality that $\pi=\pi_{1} \times \pi_{2} \times \cdots \times \pi_{n}$. 
(a) If $\left(Z_{i}, U_{i}\right)$ is trivial, then $\pi_{i}$ and hence $\pi$ is not proximal. Suppose every $\left(Z_{i}, U_{i}\right)$ is nontrivial. Then for each $i, \pi_{i}$ is proximal and $\mathbf{G}\left(X_{i}, T_{i}^{k(i)}\right)=\mathbf{G}\left(Z_{i}, U_{i}^{k(i)}\right)$. It follows that $\prod_{i=1}^{n}\left(X_{i}, T_{i}^{k(i)}\right)$ and $\prod_{i=1}^{n}\left(Z_{i}, U_{i}^{k(i)}\right)$ have the same Ellis group and $\pi$ is proximal.

(b) Suppose $\left(Z_{i}, U_{i}\right)$ is nontrivial for $1 \leqslant i \leqslant p \leqslant n$. Then $\pi_{0}=\pi_{1} \times \cdots \times \pi_{p}$ is proximal and $\tilde{\pi}_{0}$ is an isomorphism. Also, $K=\left\{T_{p+1}^{r(p+1)} \times \cdots \times T_{n}^{r(n)} \mid r(i) \in \mathbf{Z}\right\}$ is clearly a subgroup of the kernel of $\tilde{\pi}$. Let $L=\left\{T_{1}^{r(1)} \times \cdots \times T_{p}^{r(p)} \mid r(i) \in \mathbf{Z}\right\}$, which is the automorphism group of $\prod_{i=1}^{p}\left(X_{i}, T_{i}^{k(i)}\right)$ and set $\pi_{0}^{\prime}=\pi_{p+1} \times \cdots \times \pi_{n}$. Since $\tilde{\pi}=\tilde{\pi}_{0} \times \tilde{\pi}_{0}^{\prime}$ and $L \times K$ is the automorphism group of $\prod_{i=1}^{n}\left(X_{i}, T_{i}^{k(i)}\right), K$ is the kernel of $\tilde{\pi}$, and we are done.

\section{BIBLIOGRAPHY}

1. J. Auslander, Regular miminal sets. I, Trans. Amer. Math. Soc. 123 (1966), 469-479.

2. R. Chacon, Weakly mixing transformations which are not strongly mixing, Proc. Amer. Math. Soc. 22 (1969), 559-562.

3. J. P. Clay, Proximity relations in transformation groups, Trans. Amer. Math. Soc. 108 (1963), 88-96.

4. R. Ellis, Lectures on topological dynamics, Benjamin, New York, 1969.

5. H. Furstenberg, H. Keynes and L. Shapiro, Prime flows in topological dynamics, Israel J. Math. 14 (1973), 26-38.

6. S. Glasner, Proximal flows, Lecture Notes in Math., vol. 517, Springer-Verlag, 1976.

7. A. del Junco, $A$ family of counterexamples in ergodic theory, preprint.

8. H. Keynes, The structure of weakly mixing minimal transformation groups, Illinois J. Math. 15 (1971), 475-489.

9. H. Keynes and D. Newton, Real prime flows, Trans. Amer. Math. Soc. 217 (1976), 237-255.

10. N. Markley, Toplogical minimal self-joinings, Ergodic Theory and Dynamical Systems 3 (1983), 579-599.

11. D. Rudolph, An example of a measure preserving map with minimal self-joinings, and applications, $\mathrm{J}$ Analyse Math. 35 (1979), 97-122.

12. T. Wu, Notes on topological dynamics. II. Distal extension with discrete fibers and prime flows, Bull. Inst. Math. Acad. Sinica 3 (1975), 49-60.

Department of Mathematics, University of Maryland, College Park, Maryland 20742 